\title{
REGISTRATION OF OPTICAL DATA WITH HIGH-RESOLUTION SAR DATA: A NEW IMAGE REGISTRATION SOLUTION
}

\author{
T. Bahr ${ }^{\mathrm{a}}, \mathrm{X} . \mathrm{Jin}^{\mathrm{b}}$
}

\author{
a Exelis Visual Information Solutions GmbH, Gilching, Germany - thomas.bahr@exelisvis.com \\ ${ }^{\mathrm{b}}$ Exelis Visual Information Solutions, Boulder, Colorado, U.S.A - xiaoying.jin@exelisvis.com
}

KEY WORDS: Image Registration, Multisensor, Optical Data, SAR, TerraSAR-X, Pléiades-1a, Automation, HyPARE, Algorithm

\begin{abstract}
:
Accurate image-to-image registration is critical for many image processing workflows, including georeferencing, change detection, data fusion, image mosaicking, DEM extraction and 3D modeling. Users need a solution to generate tie points accurately and geometrically align the images automatically. To solve these requirements we developed the Hybrid Powered Auto-Registration Engine (HyPARE). HyPARE combines all available spatial reference information with a number of image registration approaches to improve the accuracy, performance, and automation of tie point generation and image registration. We demonstrate this approach by the registration of a Pléiades-1a image with a TerraSAR-X SpotLight image of Hannover, Germany. Registering images with different modalities is a known challenging problem; e.g. manual tie point collection is prone to error. The registration engine allows to generate tie points automatically, using an optimized mutual information-based matching method. It produces more accurate results than traditional correlation-based measures. In this example the resulting tie points are well distributed across the overlapping areas, even as the images have significant local feature differences.
\end{abstract}

\section{INTRODUCTION}

Image registration involves locating and matching tie points in the two images to be registered. In manual registration, a human operator performs these tasks visually using interactive software. The process is repetitive, laborious, tedious and prone to error. Manual registration becomes prohibitive for the large amount of data and large geographic coverage.

An automated registration solution is highly desired in a production environment. In automated registration, algorithms automatically generate tie points and align the image. However, remote sensing images have various characteristics that make automated registration difficult. Location errors may occur in the navigation and during spacecraft maneuvers. The atmospheric scattering and absorption affect the fidelity of remote sensing data. Various image characteristics due to multitemporal effects, terrain effects, different sensor modalities and different spatial resolution all make automated registration a challenging task. Automated registration in remote sensing does not always offer the needed reliability and accuracy, so often manual editing of tie points is needed.

To solve these requirements we developed the Hybrid Powered Auto-Registration Engine (HyPARE). This registration engine generates tie points automatically and accurately. It takes advantage of all the available spatial reference information to achieve the best accuracy and automation during registration. It combines a number of registration approaches to improve the reliability, accuracy, performance and automation of image registration. It is robust in the situations of images obtained from different viewing angles, in different time and seasons, with various terrain relief, and by sensors with different modalities, e. g. SAR and optical images. The algorithm is automated and optimized so that user interaction and editing is minimized or eliminated (Jin, 2012).

\section{THE HYBRID POWERED AUTO-REGISTRATION ENGINE (HYPARE)}

HyPARE combines all available spatial reference information to achieve the best accuracy and automation during registration (see Figure 1). The spatial reference information may come from the following sources (Jin, 2012):

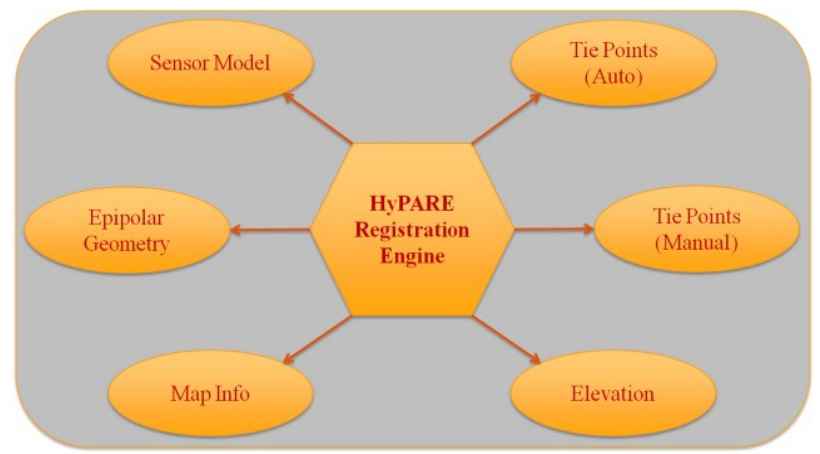

Figure 1. Key components of the Hybrid Powered AutoRegistration Engine (HyPARE)

Standard map information or RPC information of the input images establishes the approximate geometric relationship between a warp image and a base image. It reduces the search space and improves the reliability of automatic tie point generation.

For most applications, the tie point information is automatically generated with no or minimized number of outliers. The operator can manually define a few seed tie points and feed them into automatic tie point generation to improve the overall accuracy. 
Geo-location geometric constraints are used to search for and filter tie points. The images should align well in the common ground coordinate space in the orthorectified images or nadir view images; therefore, all the tie points are constrained by a global transform between the coordinates in the base image and the warp image.

RPC sensor models and elevation are used for orthorectification on-the-fly during image registration. This geometrically corrects the data to a common ground coordinate space, and achieves better accuracy in automatic tie point finding and filtering. The tie points can be automatically generated even in the image areas with big distortion during to terrain relief, such as mountainous areas.

For images taken at different viewing positions and/or angles, the images of the same scene are related by the epipolar geometry constraint. For a feature point in the first image, the corresponding point in the second image must lie on the epipolar line or curve. Two epipolar geometry models are used in geometric filtering: one is suitable for the imagery with the frame central projection, and the other is suitable for the imagery obtained with a pushbroom sensor that has RPC information.

\section{THE IMAGE REGISTRATION WORKFLOW}

For the application of the registration process we embedded the HyPARE Algorithm in a wizard guiding through file selection, automatic tie point generation, review of the tie points and preview of the warped image, and export of image registration results and tie points (Jin, 2012).

The workflow starts with the selection of a base image as the reference image and a warp image to be registered with the base image. The base image can have standard map projections or RPC information; it cannot be pixel-based, arbitrary, or a pseudo projection. The warp image does not have restrictions. If a pixel-based image or an image with pseudo projection or arbitrary projection is used as the warp image, one has to select at least three seed tie points to define the approximate relationship between the two images.

Auto tie point generation compares the gray scale values of patches of two images and tries to find joined image locations based on similarity in those gray scale value patterns. Two image matching methods are available: a correlation-based method and a mutual information-based method. The mutual information-based method is optimized for registering images with different modalities, e.g. registering radar with optical images, as demonstrated in the case study (see section 4). The normalized mutual information between the patch in the base image and the patch in the warp image is computed as the matching score. Mutual information is based on information theory and measures the mutual dependence of the two random variables (Exelis Visual Information Solutions (Ed.), 2012b). The false tie points with a low matching score are removed. The outliers are further removed by fitting and filtering by a geometric model. The operator can select the geometric model from a global transform model or two epipolar geometry based models.

If the base image or warp image includes RPC information, we recommend the use of a DEM file. The registration engine performs orthorectification on-the-fly, and generates tie points in the common ground coordinate space. The image is geometrically corrected for automatic tie point generation. Radiometric matching and geometric filtering are performed in the common ground coordinate space to improve the reliability and accuracy of tie point generation.

The automatic tie point generation algorithm is optimized to minimize or eliminate the need of manual tie point editing. However, one can choose to review, add, or delete tie points interactively. Further options within this processing step are changing the image warping and resampling parameters and previewing the image warping result.

At the end, one can export the warped image which is registered with the base image, and the tie points to be used in other applications or systems.

\section{CASE STUDY: REGISTRATION OF PLÉIADES-1A DATA WITH HIGH-RESOLUTION TERRASAR-X DATA}

For the present case study we imported a TerraSAR-X SpotLight image with $1.25 \mathrm{~m}$ GSD as the base image and a Pléiades-1a multispectral image with $0.5 \mathrm{~m}$ GSD as a warp image. The TerraSAR-X image is a geocoded ellipsoid corrected (GEC) product, i.e. a multi-look detected product (L1B). WGS84 is used as geodetic reference assuming one average terrain height. As the ellipsoid correction does not consider a DEM, the pixel location accuracy varies due to the terrain (Roth, A., Huber, M., Kosmann, D., 2004). However, as the images are located at the city area of Hannover, topography doesn't affect the location accuracy. The Pléiades-1a image was delivered as processing level primary, with RPCs.

The advantage of using geocoded high-resolution SAR images as a base for image registration is that the geocoding is performed by rigorously applying the Range and Doppler equations (Meier E., Frei U., Nüesch D., 1993). Thus, the map locations for every pixel are precisely determined.

Figure 2 shows the TerraSAR-X image with 52 automatically calculated tie points. The tie points are well distributed across the overlapping areas, even as the images have significant local feature differences. To support the automatic tie point matching, three seed tie points were selected.

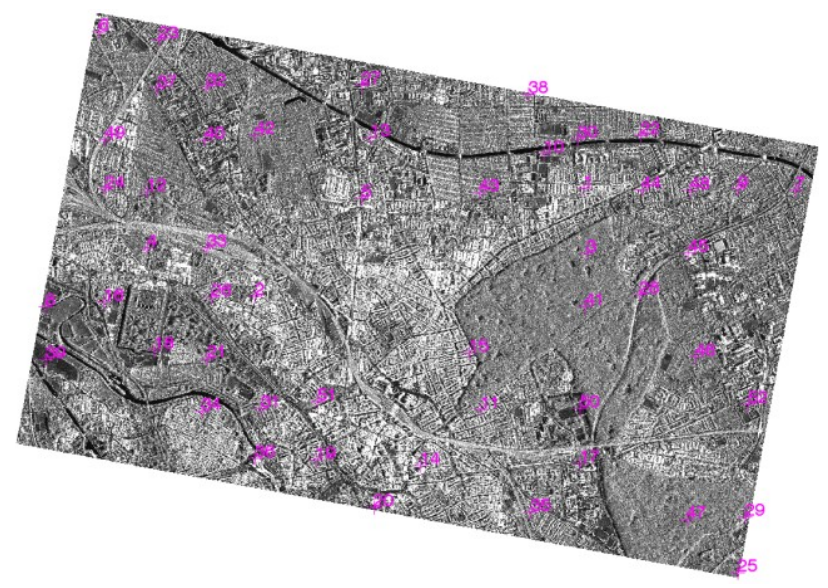

Figure 2. TerraSAR-X SpotLight image with automatically calculated tie points, Hannover. (C) CNES 2012, Distribution Astrium Services / Spot Image S.A., France, all rights reserved. 
International Archives of the Photogrammetry, Remote Sensing and Spatial Information Sciences, Volume XL-1/W1, ISPRS Hannover Workshop 2013, 21 - 24 May 2013, Hannover, Germany

Due to a total root mean square (RMS) error of $1.29 \mathrm{~m}$ the features of the warped Pléiades-1a image align well with the TerraSAR-X base image after image registration (see Figure 3 ).

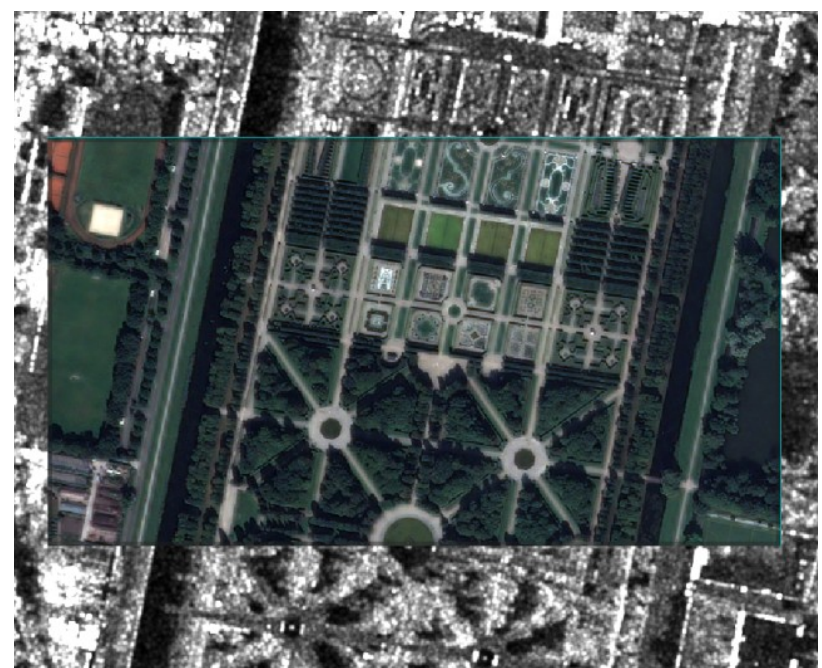

Figure 3. Pléiades-1a registration preview on TerraSAR-X SpotLight image, Hannover, Great Garden. (C) CNES 2012, Distribution Astrium Services / Spot Image S.A., France, all rights reserved.

Furthermore, this case study evidenced that the mutual information approach produces more accurate results than the traditional correlation-based measures for cross-modality image registration. Though this method takes longer to run since it is more computationally intensive, it is more robust to generate tie points from images with different modalities.

\section{CONCLUSIONS}

With the Hybrid Powered Auto-Registration Engine (HyPARE) we developed an approach to generate tie points accurately and geometrically align images of multiple sources automatically. HyPARE is robust in situations of images obtained from different viewing angles, in different time and seasons, with various terrain relief, and by sensors with different modalities. Therefore it is suitable for many applications. We demonstrated this successfully by the registration of a Pléiades-1a image with a TerraSAR-X SpotLight image of Hannover, Germany.

As the field of remote sensing keeps evolving, we are continuing our research and development on new solutions to meet the changing requirements and scope of image registration and its related projects. Future direction is to extend the automatic registration algorithm to imagery with mostly forest and desert areas, images with considerable shadows and clouds, images acquired from highly off-nadir views, etc. Future work also includes to automatically register images to LiDAR point clouds, register images to maps such as GIS vector layers (Jin, 2012).

\section{REFERENCES}

Exelis Visual Information Solutions (Ed.), 2012. ENVI Documentation, ENVI v5.0.

Jin, X., 2012. ENVI Image Registration. Exelis Visual Information Solutions Whitepaper, $26 \mathrm{p}$.
Meier E., Frei U., Nüesch D., 1993. Precise Terrain Corrected Geocoded Images. In: Schreier G. (Ed.): SAR Geocoding and Systems, Wichmann, Karlsruhe, pp. 173-186.

Roth, A., Huber, M., Kosmann, D., 2004. Geocoding of TerraSAR-X Data. In: ISPRS Proceedings, XXXV, Part B, Comm. 3, pp. 840-844. 